INTEGERS : ELECTRONIC JOURNAL OF COMBINATORIAL NUMBER THEORY 7(2)（2007), \#A22

\title{
A CHARACTERIZATION OF LATTICE-ORDERED GRAPHS
}

\author{
David Leach \\ University of West Georgia \\ cleach@westga.edu \\ Matt Walsh \\ Indian-Purdue Fort Wayne \\ walshm@ipf w. edu
}

Received: 1/27/06, Revised: 6/12/06, Accepted: 7/13/06

\begin{abstract}
A finite simple graph $G$ is said to be lattice-ordered if the poset of unlabeled induced subgraphs of $G$, ordered by inclusion, is lattice-ordered. In this paper, we prove that a graph is lattice-ordered if and only if it or its complement is complete multipartite. Furthermore, if two lattice-ordered graphs have isomorphic unlabeled induced subgraph lattices, then one can be obtained from the other via conjugations and complementations.
\end{abstract}

An induced subgraph $G[S]$ of a (finite simple) graph $G$ is defined as a set $S \subseteq V(G)$ of vertices together with edge set $\{v w: v, w \in S\}$. Throughout, we shall use $G \preceq H$ to denote that $G$ is an induced subgraph of $H$. The set of unlabeled induced subgraphs (i.e. with isomorphic induced subgraphs identified) of any graph can be partially ordered by inclusion; our ultimate goal is to determine when a graph is determined by the form of its "induced poset".

A lattice-ordered set is a poset with the property that for any two elements $a, b$ we can uniquely define $\inf \{a, b\}$ and $\sup \{a, b\}$. It is clear that the poset of labeled induced subgraphs (ordered by inclusion) is lattice-ordered. Numerous authors (see $[4,5,6,8,9]$ ) have discussed the lattices which come from the poset of connected induced subgraphs. Others (see $[1,2,3])$ have investigated posets of all simple graphs with a fixed order. We wish to investigate when the (unlabeled) induced poset of a graph is lattice-ordered. (We shall use lattice-ordered to refer to such graphs, for simplicity's sake.)

Lemma 1. Let $G$ and $H$ be graphs, and $\bar{G}$ and $\bar{H}$ their complements. If $G=H[S]$ for some vertex subset $S$, then $\bar{G}=\bar{H}[S]$. 
Lemma 2. $G$ is a lattice-ordered graph if and only if $\bar{G}$ is lattice-ordered.

Proof. We show that $G$ being lattice-ordered implies that $\bar{G}$ shares this property; the result then follows from complementation being its own inverse.

Suppose that $H_{1}, H_{2} \preceq G$; since $G$ is lattice-ordered we can find induced subgraphs $H^{+}=$ $\sup \left\{H_{1}, H_{2}\right\}$ and $H^{-}=\inf \left\{H_{1}, H_{2}\right\}$. Turning to the complements, it is clear that $\bar{H}^{+}=$ $\bar{G}\left[S^{+}\right]$contains both $\bar{H}_{1}$ and $\bar{H}_{2}$ as induced subgraphs, and that both in turn contain $\bar{H}^{-}$. If $K \preceq \bar{H}_{1}$ and $K \preceq \bar{H}_{2}$, then likewise $\bar{K} \preceq H_{1}, H_{2}$ and so $\bar{K} \preceq H^{-}$by the lattice-ordering on $G$; invoking Lemma 1 one more time, this implies that $K \preceq \bar{H}^{-}$, and so $\bar{H}^{-}=\inf \left\{\bar{H}_{1}, \bar{H}_{2}\right\}$. A similar argument shows that $\bar{H}^{+}=\sup \left\{\bar{H}_{1}, \bar{H}_{2}\right\}$

We shall require a pair of technical lemmas, one from the folklore of graph theory and the other following from the properties of lattices; we state these without proof.

Lemma 3. Either $G$ or $\bar{G}$ is connected.

Lemma 4. If $G$ is lattice-ordered and $H \preceq G$, then $H$ is lattice-ordered.

The smallest graphs that are not lattice-ordered are all of order 4 . The paw (a triangle with a pendant edge) is not lattice-ordered. To see this, note that the induced subgraphs $P_{3}$ and $K_{1} \cup P_{2}$ both feature $K_{2}$ and $K_{1} \cup K_{1}$ as induced subgraphs, and hence $\inf \left\{P_{3}, K_{1} \cup P_{2}\right\}$ is undefined. By Lemma 2, the complement of the paw is likewise not lattice-ordered. This observation, together with Lemma 4, will allow us to make use of the following theorem of Olariu from [7]:

Theorem 5. A graph is paw-free if and only if it is triangle-free or complete multipartite.

The third and final non-lattice-ordered graph of order 4 is $P_{4}$ (which is self-complementary), for the same reason given above for the paw.

One might notice that all of the other graphs of order 4 - as well as all graphs of orders 3 and below - have this in common: they are either complete multipartite graphs, or else disjoint unions of cliques (i.e. the complements of complete multipartite graphs). This might lead one (as it has led us) to guess that this is no coincidence.

We define the obvious bijection between the integer partitions of $n$ and the complete multipartite graphs on $n$ vertices by mapping the partition $\pi$ to $K_{\pi}$. If $\pi, \mu$ are integer partitions we shall use the notation $\pi \leq \mu$ to denote that $\pi$ is contained in $\mu$ (or actually, that the Ferrers diagram of $\pi$ is contained in that of $\mu$ ).

Theorem 6. A graph is lattice-ordered if and only if it or its complement is complete multipartite. 
Proof. $(\Rightarrow)$ Suppose that $G$ is a lattice-ordered graph and neither $G$ nor $\bar{G}$ is complete multipartite. From a previous observation we know that $G$ is paw-free, so Theorem 5 implies that both $G$ and $\bar{G}$ are triangle-free. The Ramsey number $R(3,3)=6$ implies that the order of $G$ is at most 5 , and checking all graphs of order $\leq 5$ yields no such $G$.

$(\Leftarrow)$ By Lemma 2 it suffices to assume that $G$ is complete multipartite and show that it is lattice-ordered; we note first that every induced subgraph of a complete multipartite is itself complete multipartite. If $\pi \leq \mu$, then clearly $K_{\pi} \preceq K_{\mu}$, and the reverse is also true; therefore, inclusion of induced subgraphs corresponds exactly with partition containment. But containment is well-known to be a lattice ordering of integer partitions, and therefore induced subgraph inclusion must be a lattice ordering on complete multipartite graphs (and hence their complements).

The correspondence between lattice-ordered graphs and integer partitions reveals a way other than complementation that two lattice-ordered graphs might yield the same lattice of induced subgraphs. Recall that two integer partitions are conjugate if their Ferrers diagrams are mirror-images of each other along the main diagonal.

Corollary 7. Let $\pi$ be an integer partition, and $\pi^{\star}$ its conjugate. Then the lattices of induced subgraphs of $K_{\pi}$ and $K_{\pi^{\star}}$ are isomorphic.

Proof. This follows inductively from the fact that for any partition $\pi$, the conjugates of the vertex-deleted subgraphs of $K_{\pi}$ are isomorphic to the vertex-deleted subgraphs of $K_{\pi^{\star}}$.

In what follows, when $G$ is a lattice-ordered graph and we talk about the conjugate of $G$ we are referring to the graph corresponding to the conjugate of the defining partition of $G$.

These two operations, complementation and conjugation, completely determine all latticeordered graphs that share the same poset structure. To prove this, we need first to construct some infrastructure.

Let $P=(S, \leq)$ be a poset. A chain in $P$ is a set $\left\{a_{1}, a_{2}, \ldots a_{k}\right\} \subseteq S$ such that $a_{i} \leq a_{i+1}$ for all positive $i<k$; the length of a chain (here $k$ ) is its size as a set. If $x, y \in S$ such that $x \leq y$, then the interval $\langle x, y\rangle$ is defined as the set $\{z \in S: x \leq z \leq y\}$. An induced chain in $P$ is an interval that is also a chain.

Let us apply these concepts to the induced-subgraph lattices. Let $\pi$ be an integer partition, and consider the induced chains of the form $\langle[1], \tau\rangle$ in the lattice of $\pi$. Notice that the partition $[2,1]$ cannot be a member of any such induced chain, since the sub-interval $\langle[1],[2,1]\rangle$ would not be a chain. Hence, $\tau$ must have one of the forms $[k]$ (for some positive integer $k$ ) or $[1, \ldots, 1]$; in the corresponding lattice-ordered graphs, these correspond to independent sets and cliques. Thus, the set of lengths of maximum induced chains of this form in the lattice of $\pi$ equals $\left\{\alpha\left(K_{\pi}\right), \omega\left(K_{\pi}\right)\right\}$, where $\alpha(G)$ and $\omega(G)$ denote the independence 
and clique numbers, respectively, of a graph $G$. Thus, given the lattice of induced subgraphs for a lattice-ordered graph, we can determine a pair of numbers containing the independence number and the clique number of the corresponding graph, though we cannot tell which is which.

Given an integer partition $\pi=\left[p_{1}, \ldots, p_{r}\right]$, let us define the following constructions. The shell $\pi_{s}$ of $\pi$ is defined as the partition $\left[p_{1}, 2,1, \ldots 1\right]$ with an equal number of parts as $\pi$. The heart $\pi_{h}$ of $\pi$ is defined as the partition $\left[p_{2}-1, p_{3}-1, \ldots, p_{r}-1\right]$ (with any parts of size 0 omitted). The shell of a partition is always defined; the heart is defined for all partitions containing $[2,2]$. (i.e. the only exceptions are those of the form $[k, 1, \ldots, 1]$.)

Lemma 8. Let $\pi$ be an integer partition with lattice $\mathcal{L}(\pi)$. If $\pi_{h}$ is well-defined, then the interval $\left\langle\pi_{s}, \pi\right\rangle$ in $\mathcal{L}(\pi)$ is isomorphic as a lattice to $\mathcal{L}\left(\pi_{h}\right)$.

Proof. Notice first that $\left\langle\pi_{s}, \pi\right\rangle$ consists precisely of all those partitions $\tau \preceq \pi$ such that $\tau_{s}=\pi_{s}$. We show that the mapping $\tau \rightarrow \tau_{h}$ defines a bijection between $\left\langle\pi_{s}, \pi\right\rangle$ and $\mathcal{L}(\pi)$. Assume that $\pi_{h}$ is well-defined. Then $\pi$ has at least two parts of size at least two, and hence $\left(\pi_{s}\right)_{h}$ is defined and equal to [1]. If $\tau \in\left\langle\pi_{s}, \pi\right\rangle$ then $\tau_{h}$ is well-defined (by transitivity), and from the definition of the heart it follows that $\tau_{h} \preceq \pi_{h}$. Suppose that $\sigma=\left[s_{1}, \ldots s_{j}\right]$ and $\tau=\left[t_{1}, \ldots, t_{k}\right]$ are in $\left\langle\pi_{s}, \pi\right\rangle$ and $\sigma_{h}=\tau_{h}$; this implies that $s_{i}=t_{i}$ for all $i$ between 2 and some $m \leq \min \{j, k\}$, and further that for all $i>m, s_{i}=1$ and $t_{i}=1$ if the partition in question has a positive $i^{\text {th }}$ part. Thus, the only places that $\sigma$ and $\tau$ can differ from each other would appear in the first row and/or column of their respective Ferrers diagrams. But this would imply that their shells are different, while we know that $\sigma_{s}=\tau_{s}$ from our first remark; hence the mapping $\tau \rightarrow \tau_{h}$ is a bijection. To show isomorphism, it suffices to note that if $\sigma, \tau \in\left\langle\pi_{s}, \pi\right\rangle$ such that $\sigma \preceq \tau$, then $\sigma_{h} \preceq \tau_{h}$.

In terms of complete multipartite graphs, the transformation of an integer partition $\pi$ to $\pi_{h}$ corresponds to deleting from $K_{\pi}$ a maximum part and a single vertex from each of the remaining parts. $\pi_{h}$ is not well-defined, then the resulting graph is null. If $G$ is a complete multipartite graph, let $G_{\odot}$ denote the result of this operation.

Theorem 9. If two lattice-ordered graphs have isomorphic unlabeled induced subgraph lattices, then one can be obtained from the other via conjugations and complementations.

Proof. Lemma 2 and Corollary 7 show that conjugations and complementations do not change the isomorphism class of the unlabeled induced subgraph lattice. We will use induction to show that given a lattice-ordered graph, these operations generate all graphs in its isomorphism class, but first we must establish the base cases.

Suppose that $G$ and $H$ are two lattice-ordered graphs with isomorphic lattices such that neither one can be obtained from the other by way of complementation or conjugation. We may assume that both $G$ and $H$ are complete multipartite graphs, and further that $\alpha(G) \leq \omega(G)$ and $\alpha(H) \leq \omega(H)$. (We may ensure both of these assumptions through taking 
complements and conjugates, if necessary.) Since the two graphs have the same lattice structure, this implies that $\alpha(G)=\alpha(H)$ and $\omega(G)=\omega(H)$.

It seems clear that $G_{\odot}$ is null if and only if $H_{\odot}$ is. Should both of these graphs be null, then we know precisely the structures of $G$ and $H$, specified completely by their clique and independence numbers, and hence $G$ and $H$ are isomorphic. We shall now proceed by induction, using these as our base cases.

Suppose that $G$ and $H$ are the minimum graphs that satisfy the conditions listed above (complete multipartite graphs with isomorphic lattices, independence numbers and clique numbers equal, and the latter being at least equal to the former); by the discussion in the last paragraph, $G_{\odot}$ and $H_{\odot}$ must both be non-null graphs. For convenience, let $G=K_{\sigma}$ and $H=K_{\tau}$. From our assumptions about clique and independence numbers of the graphs, we know that $\sigma_{s}=\tau_{s}$; this, together with the isomorphism of the lattices of $\sigma$ and $\tau$ and Lemma 8 implies that the lattices of $\sigma_{h}$ and $\tau_{h}$ must be isomorphic. The induction hypothesis then requires that these partitions be either equal or conjugate; assume the latter, since otherwise we have shown $G$ and $H$ to be isomorphic. There are now several possibilities:

- If $\alpha(G)=\omega(G)$ for the two original graphs, then $\sigma_{h}^{\star}=\tau_{h}$ implies that $\sigma^{\star}=\tau$, which satisfies our theorem.

- If $\alpha(G)<\omega(G)$ and $\alpha\left(G_{\odot}\right)>\omega(G)-1$, then $\sigma_{h}$ and $\tau_{h}$ can't be conjugates of each other, since the conjugate of $\sigma_{h}$ could not "fit" in the shell $\tau_{s}$. Hence the two graphs must be isomorphic.

- Otherwise, $G$ and $H$ must each have a part consisting of a single vertex. Delete such a vertex from each of $G$ and $H$, and the result follows from the inductive hypothesis.

This completely characterizes both which graphs are lattice-ordered, and what graphs a given lattice will correspond to (if any). One future direction for our work is to derive a similar such result for those graphs where the poset of induced subgraphs is not latticeordered; specifically, we would like to see a characterization of those posets that might represent the induced subgraphs of some graph. Additionally, we would like to determine if there is an easy set of relationships between two graphs that share their poset structure, as there is in the case of lattice-ordered graphs.

Another direction would be to apply our techniques to the more popularly studied posets of connected induced subgraphs, and the determination of which graphs are lattice-ordered in this sense. One application of our work is in the construction of several small examples of graphs where the "cisposet" is not lattice-ordered.

Additionally, we can use Theorem 6 to find a family of graphs where this poset is latticeordered: 
Corollary 10. The poset of connected induced subgraphs of a (connected) complete multipartite graph is lattice-ordered.

Proof. The poset in question is identical to the lattice of induced subgraphs, save that the chain of empty graphs is omitted. It is easy to see that the meet of two connected graphs in the original lattice will never be a collection of isolates, and thus this deletion does not disrupt the lattice-ordering.

The authors would like to thank the anonymous reviewer for his or her several helpful suggestions.

\section{References}

[1] Peter Adams, Roger B. Eggleton, and James A. MacDougall. Degree sequences and poset structure of order 9 graphs. In Proceedings of the Thirty-Fifth Southeastern International Conference on Combinatorics, Graph Theory and Computing, volume 166, pages 83-95, 2004.

[2] Peter Adams, Roger B. Eggleton, and James A. MacDougall. Structure of graph posets for orders 4 to 8. In Proceedings of the Thirty-Fifth Southeastern International Conference on Combinatorics, Graph Theory and Computing, volume 166, pages 63-81, 2004.

[3] Roger B. Eggleton and James A. MacDougall. Graph posets, spanning-universal graphs and graph coverings. Bull. Inst. Combin. Appl., 37:63-72, 2003.

[4] Michael S. Jacobson, André E. Kézdy, and Steve Seif. The poset on connected induced subgraphs of a graph need not be Sperner. Order, 12(3):315-318, 1995.

[5] André E. Kézdy and Steve Seif. When is a poset isomorphic to the poset of connected induced subgraphs of a graph? Southwest J. Pure Appl. Math., (1, July 1996):42-50 (electronic), 1996.

[6] J. Nieminen. The lattice of connected subgraphs of a connected graph. Comment. Math. Prace Mat., 21(1):187-193, 1980.

[7] Stephan Olariu. Paw-free graphs. Inform. Process. Lett., 28(1):53-54, 1988.

[8] William T. Trotter, Jr. and John I. Moore, Jr. Some theorems on graphs and posets. Discrete Math., 15(1):79-84, 1976.

[9] Jonathan Wiens and Kara L. Nance. The lattice polynomial of a graph. Ars Combin., 57:139-149, 2000. 